\title{
Estimating the Proportion of Plasmodium vivax Recurrences Caused by Relapse: A Systematic Review and Meta-Analysis
}

\author{
Robert J. Commons, ${ }^{1,2 \star}$ Julie A. Simpson, ${ }^{3}$ James Watson, ${ }^{4,5}$ Nicholas J. White, ${ }^{4,5}$ and Ric N. Price ${ }^{1,4,5}$ \\ ${ }^{1}$ Global Health Division, Menzies School of Health Research and Charles Darwin University, Darwin, Australia; ${ }^{2}$ Internal Medical Services, Ballarat \\ Health Services, Ballarat, Australia; ${ }^{3}$ Centre for Epidemiology and Biostatistics, Melbourne School of Population and Global Health, The University \\ of Melbourne, Melbourne, Australia; ${ }^{4}$ Mahidol-Oxford Tropical Medicine Research Unit (MORU), Faculty of Tropical Medicine, Mahidol University, \\ Bangkok, Thailand; ${ }^{5}$ Centre for Tropical Medicine and Global Health, Nuffield Department of Clinical Medicine, University of Oxford, Oxford, \\ United Kingdom
}

\begin{abstract}
Plasmodium vivax and Plasmodium ovale form dormant liver hypnozoites that can reactivate weeks to months following initial infection. Malaria recurrences caused by relapses are an important cause of morbidity and source of transmission. To estimate the proportions of $P$. vivax malaria recurrences caused by relapses in different geographical locations, we systematically reviewed clinical efficacy studies of uncomplicated $P$. vivax malaria, in which patients were randomized to treatment with or without radical cure primaquine regimens and were followed up for 1 year. The minimum proportion of recurrences caused by relapses was estimated for each study site by assuming primaquine prevented all relapses and did not augment blood-stage efficacy. Of the 261 studies identified, six were eligible enrolling 4,092 patients from 14 treatment arm comparisons across seven countries. Of the 2,735 patients treated with primaquine, $24.3 \%$ received low dose $(2.5$ to $<5.0 \mathrm{mg} / \mathrm{kg}$ total) and $75.7 \%$ received high-dose primaquine $(\geq 5.0 \mathrm{mg} / \mathrm{kg}$ total). The overall pooled incidence rate ratio of $P$. vivax relapses for patients treated with primaquine versus no primaquine was $0.15(95 \%$ Cl: $0.10-0.21 ; l^{2}=83.3 \%$ ), equating to a minimum of $79 \%$ of recurrences attributable to relapse. Country-specific incidence rate ratios ranged from 0.05 (95\% Cl: 0.01-0.34; one estimate) in Pakistan to 0.34 in Nepal (95\% Cl: 0.12-0.83; one estimate) and Afghanistan (95\% Cl: $0.22-0.51$; three estimates). Relapses account for a very high proportion of recurrent infections following schizontocidal treatment of acute $P$. vivax malaria across diverse geographic locations. This emphasizes the importance of implementing hypnozoitocidal treatment.
\end{abstract}

\section{INTRODUCTION}

Plasmodium vivax malaria caused an estimated 14.3 million infections in 2017. ${ }^{1}$ Unlike Plasmodium falciparum, successful treatment of blood-stage infection does not necessarily prevent recurrence. Dormant liver hypnozoites can reactivate weeks to months after the initial infection and cause recurrent parasitemia (relapse) and further episodes of clinical malaria. Recurrent malaria can also be caused either by failure to clear blood-stage infection effectively (recrudescence) or by a new infection following exposure to another infected mosquito (reinfection). ${ }^{2}$ Effective treatment of $P$. vivax malaria requires treatment of both the blood stage and persistent liver stages of the parasite; this is termed radical cure. Primaquine is the only widely available antimalarial for the treatment of dormant liver-stage parasites, which reduces the risk of relapse by over $90 \%$, with higher doses having greater anti-relapse efficacy. ${ }^{3,4}$ Because relapsing infections are important drivers of transmission of $P$. vivax malaria, ${ }^{5}$ quantification of the proportion of recurrences caused by relapse is critical to our understanding of the potential benefits of radical cure in different endemic locations.

Identifying the cause of $P$. vivax recurrence is complex. Molecular analysis of the pre- and posttreatment parasites is less informative than that in $P$. falciparum infections because relapses can be genetically similar to or different from the initial infection. ${ }^{6,7}$ Data from two clinical studies have been used to estimate the proportion of $P$. vivax parasitemias attributable to relapse in populations in Southeast Asia. A study in children aged 5-10 years in Papua New Guinea (PNG) conducted in

\footnotetext{
*Address correspondence to Robert J. Commons, Global and Tropical Health Division, Menzies School of Health Research, Charles Darwin University, P.O. Box 41096, Casuarina 0811, Australia. E-mail:
} robert.commons@gmail.com
2009-2010 compared asymptomatic patients treated with and without prolonged high-dose primaquine and estimated that $82 \%$ of $P$. vivax episodes in this high-transmission setting resulted from relapses. ${ }^{8} \mathrm{~A}$ complementary analysis of a cohort from the Thailand-Myanmar border and a separate cohort from PNG estimated relapses to cause $70 \%$ and $96 \%$ of recurrences, respectively, when assuming the anti-relapse preventive effectiveness of primaquine was $100 \%{ }^{9}$ Transmission intensities, seasonality, and the relapse periodicity of $P$. vivax vary considerably between locations. ${ }^{10}$ Thus, the proportion of recurrences attributable to $P$. vivax relapses will differ by region, location, patients' age, and season. Nevertheless, extrapolation of the benefits of radical cure in reducing $P$. vivax-associated morbidity, mortality, and transmission across the $P$. vivax-endemic world requires some estimate. Using data from anti-relapse efficacy studies, a number of which were published recently, this study aimed at providing an estimate of the proportion of $P$. vivax recurrences due to relapses in locations with different malarial endemicity.

\section{METHODS}

Data extraction. Medline, Embase, Web of Science, and the Cochrane Database of Systematic Reviews were searched for prospective clinical efficacy studies of uncomplicated $P$. vivax malaria, as described previously. ${ }^{3}$ Studies published before August 6, 2019 in any language were included if they included patients with $P$. vivax malaria treated with and without primaquine, provided that a minimum total dose of primaquine of $2.5 \mathrm{mg}$ base $/ \mathrm{kg}$ was commenced within 3 days of enrollment and administered daily thereafter in one treatment arm and that there was active follow-up for 365 days through multiple episodes of $P$. vivax parasitemia should they 
occur. Studies were excluded if they were retrospective, patients were only followed up passively, or incidence rates could not be extracted. Studies were identified by two authors (R. J. C. and R. N. P.) using the search terms in Supplemental File 1, with discrepancies resolved by discussion.

Data were extracted for each treatment site and treatment arm. When multiple primaquine regimens were used within a study, data were aggregated by total $\mathrm{mg} / \mathrm{kg}$ primaquine dose irrespective of the number of days over which the primaquine regimen was given. Extracted data included details of the study, site, duration of follow-up, schizontocidal treatment, primaquine dose and duration, patient numbers, and incidence rate of parasite recurrence over 365 days. Investigators of eligible studies in which incidence rates could not be extracted from the published article were contacted directly. Primaquine dose was defined as low if the target total dose of primaquine was $2.5-<5.0 \mathrm{mg} / \mathrm{kg}$ and high if $\geq 5.0 \mathrm{mg} / \mathrm{kg}^{4}$

Analysis. The primary outcome of the analysis was the incidence rate of $P$. vivax recurrences over 365 days following treatment for each study site, as reported in the article. The incidence rate ratio was calculated for each study site using the ratio of the incidence rate of $P$. vivax recurrences in the treatment arm with primaquine versus the treatment arm without primaquine. The minimum proportion of recurrences attributable to relapse was estimated by subtracting the incidence rate ratio from 1 . These estimates make two key assumptions: first, primaquine does not significantly improve the asexual parasite killing effect and, thus, the cure rate of the antimalarial treatment, and second, the treatment arms with primaquine were $100 \%$ effective in preventing relapses. A follow-up duration of 365 days was chosen to ensure that relapses from long-latency $P$. vivax were captured.

The pooled incidence rate ratio of recurrent $P$. vivax parasitemia in patients treated with primaquine versus those without was estimated using a Poisson regression model with random study effects. ${ }^{11,12} \mathrm{~A}$ random-effects model was used because the incidence rate ratio varies between geographic locations and studies. Subgroup estimates were calculated for primaquine dose and country. Between-study heterogeneity was quantified by the $I^{2}$ statistic. Analyses were performed using Stata version 15 (StataCorp, College Station, TX) and $R$ version 3.4.0 (R Foundation for Statistical Computing, Vienna, Austria). The study protocol was registered with PROSPERO: CRD42020158857.

\section{RESULTS}

The systematic review identified 261 studies of uncomplicated $P$. vivax malaria treatment, of which 39 (14.9\%) recruited and followed up patients for 365 days (Figure 1, Supplemental File 2). Of these, 33 studies were excluded: 30 studies did not compare the same treatment with or without primaquine, one study gave weekly primaquine, and in two studies, the total primaquine dose was $<2.5 \mathrm{mg} / \mathrm{kg}$ (Supplemental File 2). Of the remaining six studies, 224 patients from one study were excluded from an artesunate treatment arm for which there was no comparative arm with primaquine $^{13}$ and 55 patients from one study were excluded from a treatment arm because of treatment with weekly primaquine. ${ }^{14}$ In total, 4,092 patients were included in the analysis from six studies ${ }^{13-18}$ across 14 paired treatment arms

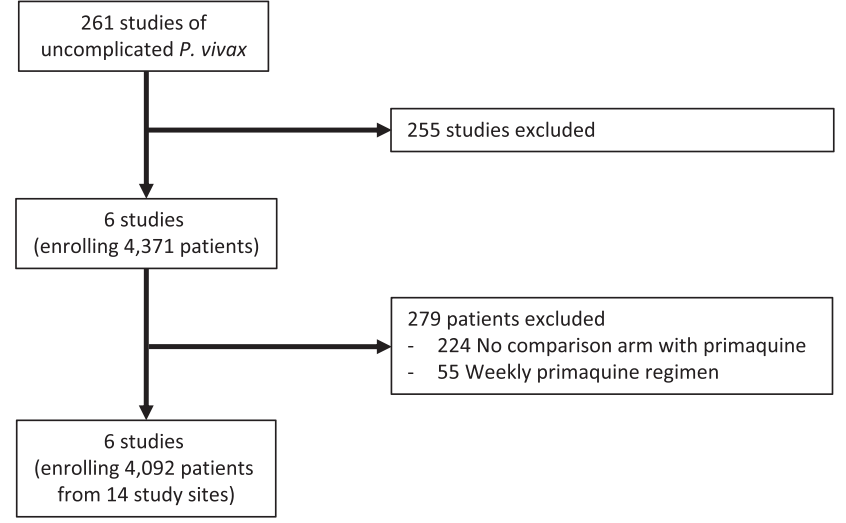

Figure 1. Flow diagram. Reasons for study exclusion are detailed in Supplemental File 2.

with and without primaquine (Table 1). Data to calculate incidence rates were extracted from four studies directly, with additional data on incidence rates provided by investigators for two studies.

Overall, 1,018 patients were enrolled in Afghanistan (three paired treatment arms), 978 in Ethiopia (four paired treatment arms), 1,000 in Indonesia (two paired treatment arms), 420 from the Thailand-Myanmar border (one paired treatment arm), 325 in Vietnam (two paired treatment arms), 206 in Nepal (one paired treatment arm), and 145 in Pakistan (one paired treatment arm). The blood schizonticide administered was chloroquine in 2,983 (72.9\%) patients, dihydroartemisininpiperaquine in $1,000(24.4 \%)$ patients, and artemetherlumefantrine in 192 (4.7\%) patients. A low-dose primaquine regimen $(0.25 \mathrm{mg} / \mathrm{kg}$ for 14 days) was given to 665 (24.3\%) patients from five treatment arms, and a high-dose regimen ( $0.5 \mathrm{mg} / \mathrm{kg}$ for 14 days or $1 \mathrm{mg} / \mathrm{kg}$ for 7 days) was given to $2,070(75.7 \%)$ patients from nine treatment arms.

Compared with patients who were not treated with primaquine, the incidence rate ratios of $P$. vivax recurrences for patients treated with primaquine ranged from 0.05 to 0.39 , with an overall pooled incidence rate ratio of $0.15(95 \% \mathrm{Cl}$ : $0.10-0.21 ; l^{2}=83.3 \% ; 14$ estimates; Figure 2), consistent with $85 \%$ of recurrences being due to relapses, or conservatively $79 \%$ based on the upper $\mathrm{Cl}$.

Patients treated with a low dose of primaquine had a pooled incidence rate ratio of $0.24\left(95 \% \mathrm{Cl}: 0.16-0.34 ; I^{2}=56.7 \%\right.$; five estimates), and patients treated with a high dose of primaquine had a pooled incidence rate ratio of $0.12(95 \% \mathrm{Cl}$ : $0.08-0.18 ; l^{2}=79.8 \%$; nine estimates). Country-specific estimates ranged from 0.05 (95\% Cl: 0.01-0.34; one estimate) in Pakistan to 0.34 in Nepal (95\% Cl: 0.12-0.83; one estimate) and also 0.34 in Afghanistan (95\% Cl: 0.22-0.51; three estimates), suggesting a minimum of $66 \%$ of recurrences were caused by relapse (Figure 3 ).

Methodological factors potentially contributing to bias are presented in Table 1. Most studies re-treated patients with recurrence with the same treatment that they were initially allocated. However, in one study, patients with recurrence were re-treated with unsupervised primaquine, after initial treatment with supervised primaquine. ${ }^{16}$ Because this will have potentially led to attenuation of the estimated incidence rate ratio, the analysis was repeated excluding this study. The overall pooled incidence rate ratio of 0.14 (95\% Cl: $0.09-0.21$; 
COMMONS AND OTHERS

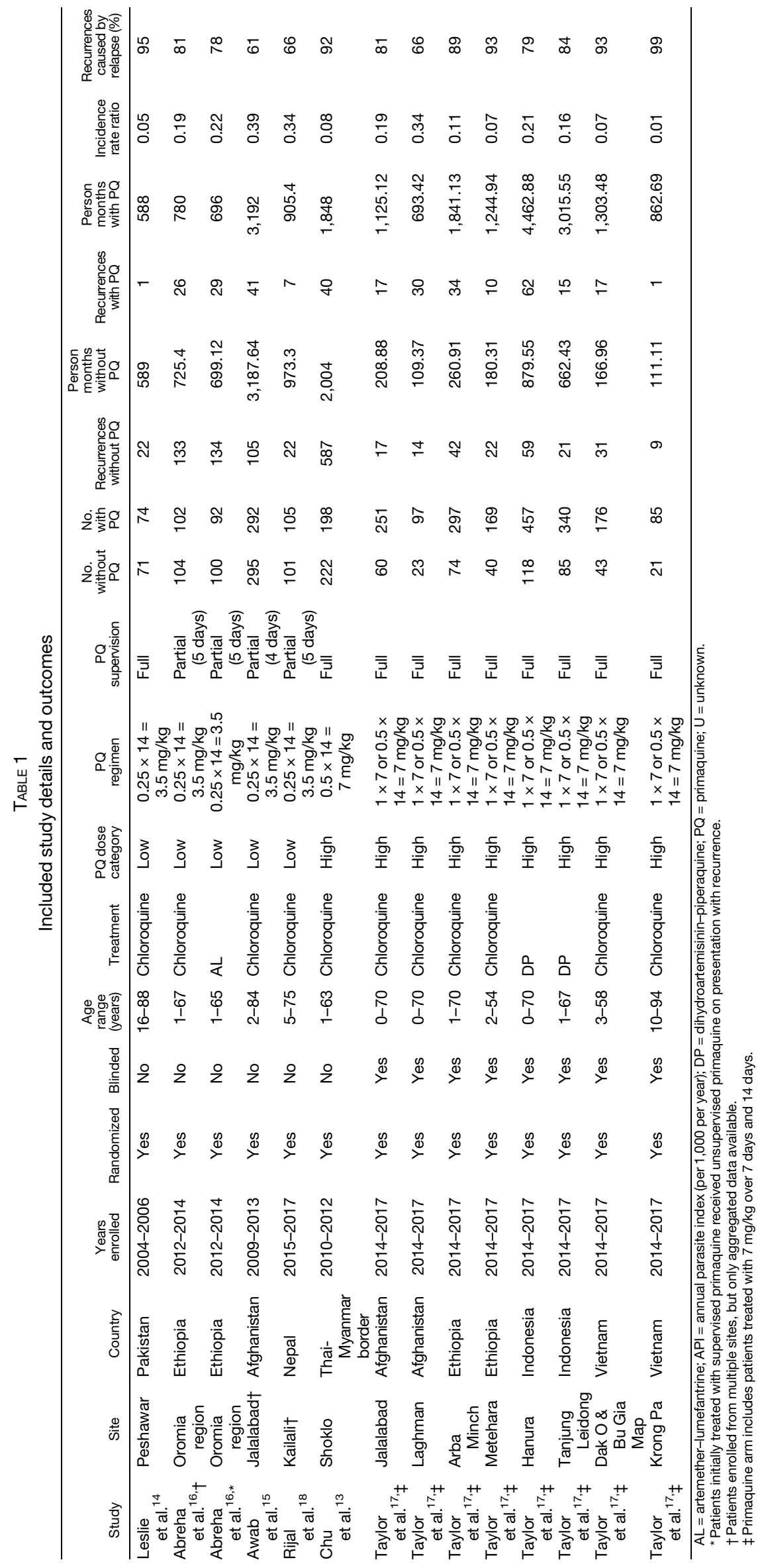




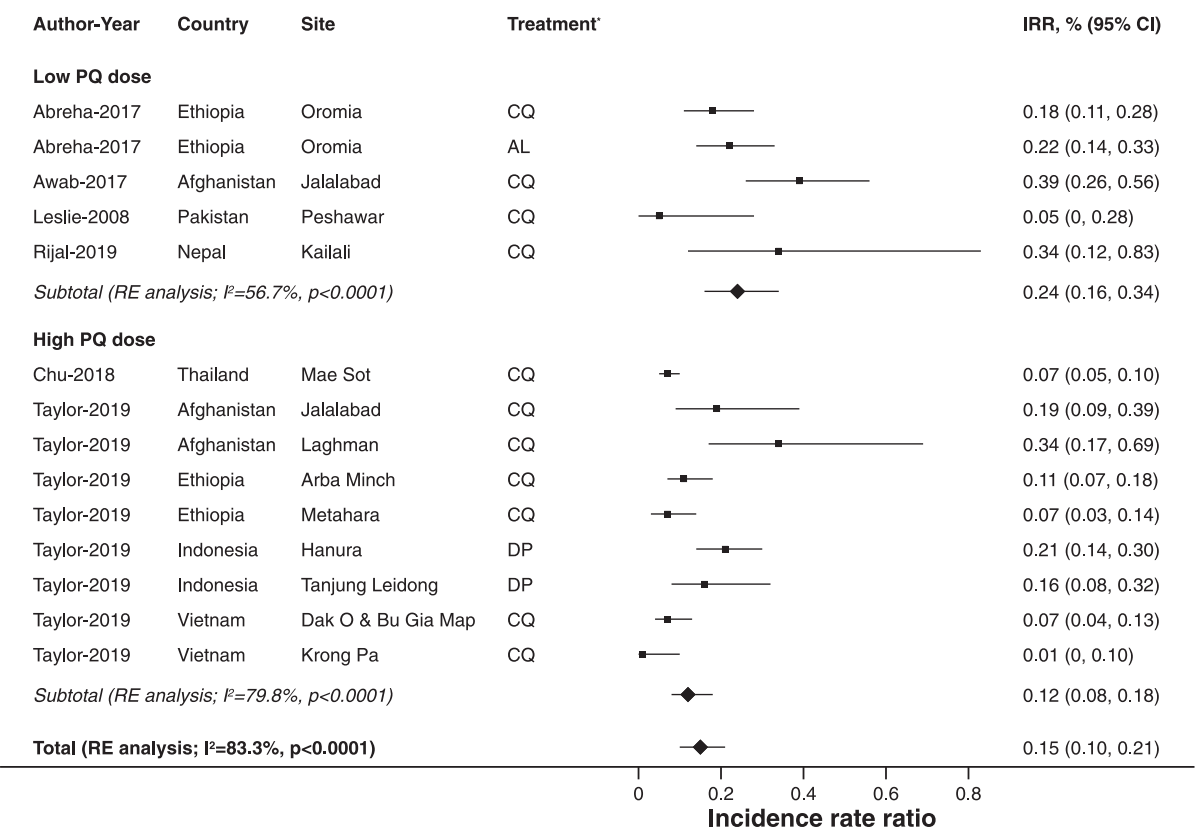

FIGURE 2. Pooled estimates of the incidence rate ratios of $P$. vivax recurrences within 365 days of follow-up comparing treatment with primaquine with no primaquine by total primaquine dose administered (low dose 2.5 to $<5 \mathrm{mg} / \mathrm{kg}$, high dose $\geq 5 \mathrm{mg} / \mathrm{kg}$ ). AL = artemether-lumefantrine; CQ = chloroquine; DP = dihydroartemisinin-piperaquine; IRR = incidence rate ratio. * Blood schizontocidal treatment.

$I^{2}=84.8 \%$; 12 estimates) was consistent with $86 \%$ of recurrences resulting from relapses.

\section{DISCUSSION}

In this meta-analysis, the minimum proportion of $P$. vivax recurrences related to relapse was estimated across several $P$. vivax-endemic countries from data derived from antirelapse clinical trials. Under the reasonable assumption that the only effect of primaquine was to eliminate hypnozoites, overall, $85 \%$ of recurrences were estimated to be related to relapses, with a minimum country-based estimate of $66 \%$ recorded in Afghanistan and Nepal. The study highlights a consistently high burden of $P$. vivax episodes attributable to relapse across diverse endemic settings. Previous estimates are based on clinical trials conducted in Southeast Asia and are thus potentially biased by the high relapse rates of $P$. vivax malaria in these areas and likely high hypnozoite burdens. ${ }^{1,10}$

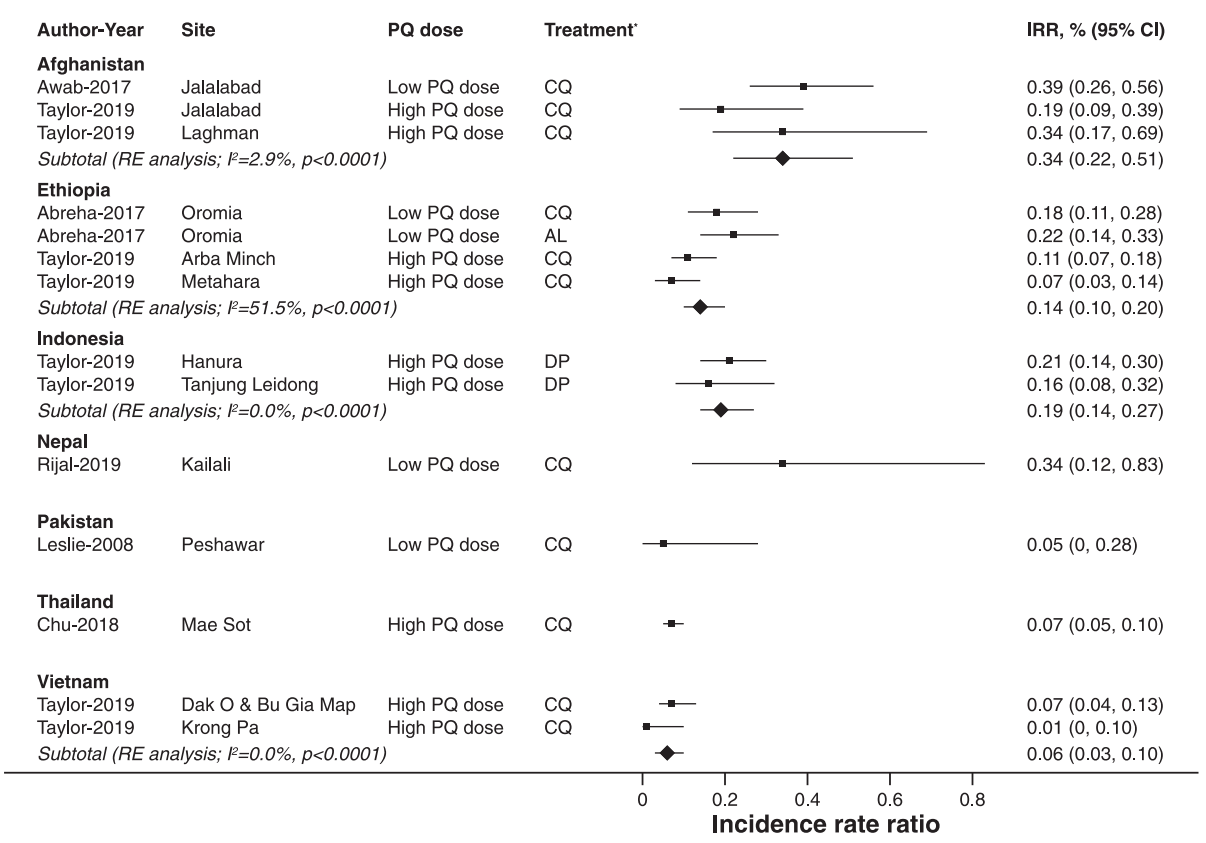

FIGURE 3. Pooled estimates of incidence rate ratios of $P$. vivax recurrences within 365 days of follow-up comparing treatment with primaquine with no primaquine by country. $\mathrm{AL}=$ artemether-lumefantrine; $\mathrm{CQ}=$ chloroquine; $\mathrm{DP}=$ dihydroartemisinin-piperaquine; IRR = incidence rate ratio *Blood schizontocidal treatment. 
The high proportion of recurrences caused by relapse in our study supports a recent population model of recurrent parasitemia on the Thailand-Myanmar border, in which the cause of recurrent infections was estimated probabilistically by incorporating molecular information on genetic relatedness and time to recurrence. ${ }^{19}$ In this location, $95 \%$ of recurrent $P$. vivax episodes following treatment with chloroquine were attributed to relapse.

We estimated the minimum proportion of recurrent $P$. vivax episodes related to relapse within a year of an initial infection, without ascribing the origin of the initial infection as being a relapse or a new infection. This differs from the earlier study by Robinson et al. ${ }^{8}$ which estimated the proportion of all $P$. vivax episodes within a population caused by relapse by following up a cohort of patients after treatment with a very high dose of primaquine (10 mg/kg total dose) to clear residual hypnozoites.

To derive a lower bound on the proportion of recurrence due to relapse, we assumed that primaquine treatment was $100 \%$ effective and, thus, that any recurrences following primaquine treatment resulted from background reinfection. In reality, this is not the case, as highlighted by clinical trials in which patients are not re-exposed to reinfection, receive a full course of radical treatment, and yet continue to have recurrent infections. ${ }^{20,21}$ Relapses following primaquine treatment are related to host, parasite, and drug factors. Host factors include treatment adherence, innate and acquired immunity, and cytochrome P450 2D6 (CYP2D6) polymorphisms. Drug factors include the dose of primaquine and pharmacokineticpharmacodynamic interactions. ${ }^{22,23}$ Parasite factors include hypnozoite burden, latency, and intrinsic drug susceptibility. ${ }^{10}$ Hence, the optimal dose for primaquine may vary markedly between different endemic regions. ${ }^{24}$ The comparative benefit of low- and high-dose primaquine regimens and how this differs across different endemic settings remains unclear. As expected, our analysis found that the pooled incidence rate ratios were lower following high-dose primaquine $(0.12)$ than following low-dose primaquine (0.24), consistent with antirelapse efficacy being related to primaquine dose. In both cases, our estimates of the proportion attributable to relapses are conservative, as our assumption of $100 \%$ primaquine antirelapse efficacy will lead to an underestimation of the relapseattributable proportion of recurrences.

Host CYP2D6 loss of function polymorphisms are associated with reduced primaquine efficacy, ${ }^{23}$ and their prevalence also differs between populations. ${ }^{25}$ Similar to the underdosing of primaquine, inclusion of patients with reduced primaquine metabolism will have led to reduced efficacy. Thus, our assumption of $100 \%$ primaquine anti-relapse efficacy will not have been correct for patients with some CYP2D6 polymorphisms, leading us to further underestimate the relapseattributable proportion of recurrences.

The known geographic differences between CYP2D6 polymorphisms and primaquine anti-relapse efficacy, combined with differences in transmission over time, may have contributed to the identified heterogeneity between study sites in the current study. In addition, variations in supervision of primaquine dosing and differing durations of primaquine treatment will lead to variations in adherence and, thus, total primaquine dose administered.

The use of primaquine treatment as the primary comparator arm also assumes that the risk of recrudescence is similar in patients treated with and without primaquine. However, primaquine has blood schizontocidal activity that reduces early recurrent infection likely from both recrudescence and relapse. ${ }^{26,27}$ In locations with reduced chloroquine susceptibility, the number of recrudescences may have been lower in the primaquine treatment arms, resulting in an overestimation of the proportion of recurrences attributable to relapse. However, this is likely to have had a minimal impact on the analysis because the Indonesian study, the only one in this analysis with highly chloroquine-resistant $P$. vivax, used dihydroartemisinin-piperaquine as the blood schizontocide.

Chloroquine and piperaquine have long half-lives that may suppress the first relapse, ${ }^{13}$ and this may have reduced the number of recurrent infections in the treatment arm without primaquine, thus underestimating the proportion of recurrences attributable to relapse.

Restricting the inclusion criteria to studies with 365 days of follow-up ensured that relapses from long-latency $P$. vivax were detected and thereby enabled direct comparisons of incidence rates. However, limiting study eligibility risks selection bias and a reduction in the generalizability of our analysis.

In summary, in all studies included in this analysis, a very high proportion of $P$. vivax malaria recurrences were attributable to relapse, and this was consistent across a diverse range of $P$. vivax-endemic settings. As progress toward malaria elimination begins to falter, ${ }^{28}$ our study once again highlights that hypnozoites are the single most important target to change the current trajectory and reduce the $P$. vivax prevalence. Recent advances in glucose-6-phosphate dehydrogenase (G6PD) diagnostics ${ }^{29}$ and novel regimens for radical cure such as high-dose short-course primaquine ${ }^{17,30}$ and single-dose tafenoquine ${ }^{31,32}$ offer important alternative approaches for the radical cure of $P$. vivax malaria. The high proportion of recurrences attributable to relapse suggests that if implemented safely and effectively, improved strategies to ensure radical cure would have a significant public health impact. This will be critical in achieving the ambitious malaria elimination targets in the Asia-Pacific, Americas, and Horn of Africa.

Received March 12, 2020. Accepted for publication April 21, 2020. Published online June 8, 2020.

Note: Supplemental files appear at www.ajtmh.org.

Financial support: N. J. W. is a Wellcome Trust Principal Fellow, and R. N. P. is a Wellcome Trust Senior Fellow in Clinical Science (200909). J.A. S. is funded by an Australian NHMRC Senior Research Fellowship 1104975. This work was supported by the Australian Centre for Research Excellence on Malaria Elimination (ACREME), funded by the NHMRC of Australia (1134989).

Authors' addresses: Robert J. Commons, Internal Medical Services, Ballarat Health Services, Ballarat, Australia, E-mail: robert.commons@ gmail.com. Julie A. Simpson, Centre for Epidemiology and Biostatistics, Melbourne School of Population and Global Health, The University of Melbourne, Melbourne, Australia, E-mail: julieas@ unimelb.edu.au. James Watson and Nicholas J. White, MahidolOxford Tropical Medicine Research Unit (MORU), Faculty of Tropical Medicine, Mahidol University, Bangkok, Thailand, E-mails: james@tropmedres.ac and nickwdt@tropmedres.ac. Ric N. Price, Global Health Division, Menzies School of Health Research and Charles Darwin University, Casuarina, Australia, E-mail: rprice@ menzies.edu.au.

This is an open-access article distributed under the terms of the Creative Commons Attribution (CC-BY) License, which permits unrestricted use, distribution, and reproduction in any medium, provided the original author and source are credited. 


\section{REFERENCES}

1. Battle KE et al., 2019. Mapping the global endemicity and clinical burden of Plasmodium vivax, 2000-17: a spatial and temporal modelling study. Lancet 394: 332-343.

2. White NJ, 2002. The assessment of antimalarial drug efficacy. Trends Parasitol 18: 458-464.

3. Commons RJ et al., 2018. The effect of chloroquine dose and primaquine on Plasmodium vivax recurrence: a WorldWide Antimalarial Resistance Network systematic review and individual patient pooled meta-analysis. Lancet Infect Dis 18: 1025-1034.

4. John GK, Douglas NM, von Seidlein L, Nosten F, Baird JK, White NJ, Price RN, 2012. Primaquine radical cure of Plasmodium vivax: a critical review of the literature. Malar J 11: 280.

5. Douglas NM, John GK, von Seidlein L, Anstey NM, Price RN, 2012. Chemotherapeutic strategies for reducing transmission of Plasmodium vivax malaria. Adv Parasitol 80: 271-300.

6. Imwong $\mathrm{M}$ et al., 2007. Relapses of Plasmodium vivax infection usually result from activation of heterologous hypnozoites. $J$ Infect Dis 195: 927-933.

7. Price RN, Auburn S, Marfurt J, Cheng Q, 2012. Phenotypic and genotypic characterisation of drug-resistant Plasmodium vivax. Trends Parasitol 28: 522-529.

8. Robinson LJ et al., 2015. Strategies for understanding and reducing the Plasmodium vivax and Plasmodium ovale hypnozoite reservoir in Papua New Guinean children: a randomised placebo-controlled trial and mathematical model. PLoS Med 12: e1001891.

9. Adekunle Al, Pinkevych M, McGready R, Luxemburger C, White LJ, Nosten F, Cromer D, Davenport MP, 2015. Modeling the dynamics of Plasmodium vivax infection and hypnozoite reactivation in vivo. PLoS Negl Trop Dis 9: e0003595.

10. White NJ, 2011. Determinants of relapse periodicity in Plasmodium vivax malaria. Malar J 10: 297.

11. Stijnen T, Hamza TH, Ozdemir P, 2010. Random effects metaanalysis of event outcome in the framework of the generalized linear mixed model with applications in sparse data. Stat Med 29: 3046-3067.

12. Spittal MJ, Pirkis J, Gurrin LC, 2015. Meta-analysis of incidence rate data in the presence of zero events. BMC Med Res Methodol 15: 42

13. Chu CS et al., 2018. Comparison of the cumulative efficacy and safety of chloroquine, artesunate, and chloroquine-primaquine in Plasmodium vivax malaria. Clin Infect Dis 67: 1543-1549.

14. Leslie T, Mayan I, Mohammed N, Erasmus P, Kolaczinski J, Whitty CJ, Rowland M, 2008. A randomised trial of an eight-week, once weekly primaquine regimen to prevent relapse of Plasmodium vivax in Northwest Frontier Province, Pakistan. PLoS One 3: e2861.

15. Awab GR, Imwong M, Bancone G, Jeeyapant A, Day NPJ, White NJ, Woodrow CJ, 2017. Chloroquine-Primaquine versus Chloroquine Alone to Treat Vivax Malaria in Afghanistan: An Open Randomized Superiority Trial. Am J Trop Med Hyg 97: 1782-1787.
16. Abreha T et al., 2017. Comparison of artemether-lumefantrine and chloroquine with and without primaquine for the treatment of Plasmodium vivax infection in Ethiopia: a randomized controlled trial. PLoS Med 14: e1002299.

17. Taylor WRJ et al., 2019. Short-course primaquine for the radical cure of Plasmodium vivax malaria: a multicentre, randomised, placebo-controlled non-inferiority trial. Lancet 394: 929-938.

18. Rijal KR et al., 2019. Efficacy of primaquine in preventing shortand long-latency Plasmodium vivax relapses in Nepal. J Infect Dis 220: 448-456.

19. Taylor AR et al., 2019. Resolving the cause of recurrent Plasmodium vivax malaria probabilistically. Nat Commun 10: 5595.

20. Nelwan EJ et al., 2015. Randomized trial of primaquine hypnozoitocidal efficacy when administered with artemisinincombined blood schizontocides for radical cure of Plasmodium vivax in Indonesia. BMC Med 13: 294.

21. Sutanto l et al., 2013. Randomized, open-label trial of primaquine against vivax malaria relapse in Indonesia. Antimicrob Agents Chemother 57: 1128-1135.

22. Schmidt LH, Fradkin R, Vaughan D, Rasco J, 1977. Radical cure of infections with Plasmodium cynomolgi: a function of total 8-aminoquinoline dose. Am J Trop Med Hyg 26: 1116-1128.

23. Baird JK et al., 2018. Association of impaired cytochrome P450 2D6 activity genotype and phenotype with therapeutic efficacy of primaquine treatment for latent Plasmodium vivax malaria. JAMA Netw Open 1: e181449.

24. WHO, 2015. Guidelines for the Treatment of Malaria, 3rd edition. Geneva, Switzerland: World Health Organization.

25. GaedigkA, Sangkuhl K, Whirl-Carrillo M, Klein T, Leeder JS, 2017. Prediction of CYP2D6 phenotype from genotype across world populations. Genet Med 19: 69-76.

26. Pukrittayakamee S, Chantra A, Simpson JA, Vanijanonta S, Clemens R, Looareesuwan S, White NJ, 2000. Therapeutic responses to different antimalarial drugs in vivax malaria. Antimicrob Agents Chemother 44: 1680-1685.

27. Pukrittayakamee S, Vanijanonta S, Chantra A, Clemens R, White NJ, 1994. Blood stage antimalarial efficacy of primaquine in Plasmodium vivax malaria. J Infect Dis 169: 932-935.

28. World Health Organization, 2018. World Malaria Report 2018. Geneva, Switzerland: WHO.

29. Pal S, Bansil P, Bancone G, Hrutkay S, Kahn M, Gornsawun G, Penpitchaporn P, Chu CS, Nosten F, Domingo GJ, 2019. Evaluation of a novel quantitative test for glucose-6-phosphate dehydrogenase deficiency: bringing quantitative testing for glucose-6-phosphate dehydrogenase deficiency closer to the patient. Am J Trop Med Hyg 100: 213-221.

30. Chu CS et al., 2018. Chloroquine versus dihydroartemisininpiperaquine with standard high-dose primaquine given either for 7 days or 14 days in Plasmodium vivax malaria. Clin Infect Dis 68: 1311-1319.

31. Lacerda MVG et al., 2019. Single-dose tafenoquine to prevent relapse of Plasmodium vivax malaria. $N$ Engl J Med 380: 215-228.

32. Llanos-Cuentas A et al., 2019. Tafenoquine versus primaquine to prevent relapse of Plasmodium vivax malaria. N Engl J Med 380: 229-241. 